Final version published as:

A. Biedermann, J. Curran, Drawbacks in the scientification of forensic

science, Forensic Science International, Volume 245, December 2014, Pages

e38-e40, ISSN 0379-0738, http://dx.doi.org/10.1016/j.forsciint.2014.10.012.

(http://www.sciencedirect.com/science/article/pii/S0379073814004186)

\title{
Letter to the editor:
}

\section{Drawbacks in the scientification of forensic science}

\begin{abstract}
This letter to the Editor comments on the article On the limitations of probability in conceptualizing pattern matches in forensic science by P. T. Jayaprakash (Forensic Science International, [10]).

4 Keywords: Probability, Statistics, Forensic science
\end{abstract}

\section{Dear Editor,}

$6 \quad$ In his letter On the limitations of probability in conceptualizing pattern matches in forensic science, Jayaprakash [10, p. 3] opines that, in "(...) physical matching, (...) interpretation using statistics is out of the question (...)". In partic-

8 ular, the author asserts that "[i]t is a reality in mathematics that probability, a technique solely relying on numerical data, is unsuitable to deal with the continuum of points in the interval $[0,1](\ldots)$ ". His main conclusion is that "(..) the 10 inability to evaluate pattern matching on a probabilistic basis is a scientific fact (...)".

This position is incorrect on both theoretical and applied grounds. First and foremost, probability theory is of

12 course perfectly well suited to deal with continuous variables [e.g., 17]. If that was not true, then forensic science - if not the whole of science - as we know it today would not be possible. Second, on a practical account, morphological

14 features (e.g., the shape of handwritten characters, as demonstrated in research published elsewhere in this journal [e.g., 14]) can be described, and hence studied, on a quantitative level using statistical methodology.

${ }_{16}$ Both these points are scientific realities, and disregarding them amounts to dismissing a scientific position, which is problematic. The fact that demonstrably wrong assertions are being published in a peer-reviewed journal such as

${ }_{18}$ Forensic Science International is a cause of concern. It reveals, and exemplifies, the more fundamental problem that there are forensic commentators who continue to argue that their field of activity is so specialized and distinctive that

20 it cannot be approached with the established concepts that the traditional scientific disciplines provide us. This is a contentious widely accepted belief that is typically promoted by many proponents in the so-called 'identification'

22 or 'individualization' disciplines. Their mode of thinking leads practitioners to endorse the vague, and unnecessary, notions of uniqueness and individuality [5,6] as grounds for categorical claims of (common) source, or as noted

24 by Jakaprakash himself, “(...) capable of generating conclusive proof on origin (...)” [10, p. 5]. This phrase is a combination of authoritative words devoid of any argument or proof. It reiterates the appeal to faith [19] that is needed

${ }_{26}$ when one oversteps the range of conclusions that can justifiably be made through defensible modes of reasoning and analysis.

28 Jayaprakash argues - with reference to statistical textbooks from the 1960s and 1980s - that statistics is applicable only in situations where numerical data are available (i.e., that statistics is unsuitable for qualitative studies and the

30 study of individual items) and that it bears a risk of being used to support any 'pet theory'. Again, this is only opinion, but not an argument, and a closer look at the current state-of-the-art in the field reveals a quite different and indeed

32 much less restricted view. For the purpose of illustration, let us go even further back in history, such as the 1950s:

"Years ago a statistician might have claimed that statistics deals with the processing of data. As a result of relatively recent formulations of statistical theory, today's statistician will be more likely to say that statistics is concerned with decision making in the face of uncertainty. Its applicability ranges from almost

36 all inductive sciences to many situations that people face in everyday life when it is not perfectly obvious what they should do." [4, p. 1]

This perspective has been fostered by many devoted scholars through decades of fundamental research and has crys2 tallized our current understanding of statistics as the study of reasonable reasoning and decision making in the face of 
uncertainty. It is the philosophy of statistics as circumscribed by Lindley:

$4 \quad$ "The philosophical position adopted here is that statistics is essentially the study of uncertainty and that the statistician's role is to assist workers in other fields, the clients, who encounter uncertainty in their work. In practice, there is a restriction in that statistics is ordinarily associated with data; and it is the link between the uncertainty, or variability, in the data and that in the topic itself that has occupied statisticians. Some writers even restrict the data to be frequency data, capable of near-identical repetition. Uncertainty, away from data, has rarely been of statistical interest. Statisticians do not have a monopoly of studies of uncertainty. Probabilists discuss how randomness in one part of a system affects other parts. Thus the model for a stochastic process provides predictions about the data that the process will provide. The passage from process to data is clear; it is when we attempt a reversal and go from data to process that difficulties appear (...), commonly called inference, and the action that it might generate.” [12, p. 294]

14 In all of this, the availability of quantitative data is surely an asset, but it is not a necessary requirement for enabling coherent reasoning. Probabilistic reasoning can also be conducted on a qualitative level [e.g., 16, 21], which is a 16 relevant topic for forensic scientists [2].

Statistics therefore provides us with the full package that we need to address the real problems in forensic science 18 on an operational level. On the one hand, we have probability theory that allows us to reason coherently in situations characterized by uncertainty. On the other hand, we have decision theory, which instructs us how to use coherently

20 informed beliefs in action. As shown elsewhere [1,20], we can state this more explicitly as follows: probability theory allows us to revise our belief about propositions of interest (e.g., propositions of common source), based on

22 results of comparative examinations, which results in an indication as to whether (and if so, to what extent) or not our uncertainty about those propositions ought to be reduced; decision theory instructs us in how to make a decision based

24 on those coherently informed beliefs, that is a decision about whether or not we ought to conclude that two compared items come from a common source.

26 Can statistics be used to support a 'pet theory' as argued by Jayaprakash, and amount to “(...) imposing unscientific application of statistics for the scholastic pretence of achieving a stamp of authority" [10, p. 6] ? This is a delusive

28 question. Without doubt, it is important to enquire about the meaningful use of statistics, but where this might not be the case, it would not be the fault of statistics, only that of poor application by an errant scientist. The very pet theory 30 is to claim the contrary.

The bottom-line recommendation made by Jayaprakash is to conduct “(...) further research on the minimum area 32 requirements for point to point qualitative comparison of the continuum patterns relevant for forensic investigation (...)" $[10$, p. 6]. Personally, it seems contrary to the nature of science to encourage scientists to make the minimum

34 amount of effort to satisfy a requirement. It is also interesting to note that this call is actually self-defeating for the author because, for any research that may be conducted in the area, the ultimate and fundamental question will be:

36 'How is one to use the research output in an argumentatively sound way?'. The single essential answer to this is that the topic is one in scientific reasoning [9], of which statistics is a special case. If it is our common aim, including that

38 of this journal, to promote the scientification of forensic science, then it is hard to understand why there are proponents who opine that they could get away without statistics, a troubling concern also raised recently elsewhere [7].

40 Jayaprakash's discourse seems to suggest that the choice of probability theory is merely one of convenience that one may endorse if one wishes to, depending on whether one feels comfortable with the topic. However, this 42 understanding misconstrues the normative nature of the theory [3]. Let us be clear about this aspect: "The key point here is that the ways in which statements of uncertainty can be combined are by no means arbitrary. One cannot sit

44 down and think up apparently reasonable rules. The only ones are the three [laws of probability] we have discussed: no more, no less. Some workers have suggested other types of combination (...). These ideas are false, because

46 one is not free to engage in the intellectual exercise of law creation. The laws are forced upon you. It is a case of the inevitability of probability. The laws ensure that several statements of uncertainty cohere" [11, p. 37, text in 48 rectangular brackets added by the author].

To the best of our knowledge, no forensic scientist has come up yet with a cogent argument to overturn the 50 fundamental tenets of probability theory. Rather, what seems to be the case in forensic science is what Lindley has seen as a prevalent position among statisticians: "The general attitude of statisticians to the Bayesian argument is to turn their heads the other way. You put the argument but most of them don't come back with any real response. In my 2 view, they can't come back with a response, but they do not even try. The most distinguished of statisticians just say 
"Yes, yes," then turn their heads away and carry on as if nothing had happened. This makes them appear polite." [18, 4 p. 312]

Perhaps Jayaprakash seeks to suggests that probability theory may be difficult to apply. This may indeed be a 6 relevant observation, but it is questionable whether this should serve as criterion for choosing or not probability as a framework for reasoning, and hence judging its adequacy. As noted concisely by Friedman: "If applied to take into

8 account all the information we have about a situation, Bayesian analysis requires unrealistically complex calculations, but this does not suggest a problem with the theory. On the contrary, the complexity is in the world surrounding us,

10 and the theory would have limited value if it could not in principle represent that complexity. Probability is a flexible template. It can take into account as much complexity as its user is able to handle" [8, p. 1818]. Jayaprakash's call

12 for “(...) acknowledging the limitations of probability (...)" [10, p. 6] thus misses the point, and hits the wrong target. Instead, what is needed to make forensic practice a science is acknowledgement of the need for an interdisciplinary

14 collaboration with the inference and decision sciences - statistics in particular - that will help us work on (i) common grounds for understanding uncertainty and its handling through probability [13], (ii) the good practice of operational

16 probability elicitation [15], and (iii) the mastery of the fundamentals of probability as a basis for decision-making [11].

\section{References}

[1] Biedermann, A., Bozza, S. and Taroni, F. [2008], 'Decision theoretic properties of forensic identification: underlying logic and argumentative implications', Forensic Science International 177, 120-132.

[2] Biedermann, A. and Taroni, F. [2006], 'Bayesian networks and probabilistic reasoning about scientific evidence when there is a lack of data', Forensic Science International 157, 163-167.

[3] Biedermann, A., Taroni, F. and Aitken, C. [2014], 'Liberties and constraints of the normative approach to evaluation and decision in forensic science: a discussion towards overcoming some common misconceptions', Law, Probability and Risk 13, 181-191.

[4] Chernoff, H. and Moses, L. E. [1959], Elementary Decision Theory, John Wiley \& Sons, New York.

26 [5] Cole, S. A. [2009], 'Forensics without uniqueness, conclusions without individualization: The new epistemology of forensic identification', Law, Probability and Risk $\mathbf{8}, 233-255$.

28 [6] Cole, S. A. [2014], 'Individualization is dead, long live individualization! Reforms of reporting practices for fingerprint analysis in the United States', Law, Probability and Risk 13, 117-150.

30 [7] Curran, J. M. [2013], 'Editorial: Is forensic science the last bastion of resistance against statistics?', Science $\mathcal{E}$ Justice 53, 251-252.

[8] Friedman, R. D. [1996], 'Assessing evidence', Michigan Law Review 94, 1810-1838.

32 [9] Howson, C. and Urbach, P. [2005], Scientific Reasoning: The Bayesian Approach, 3rd edn, Open Court.

[10] Jayaprakash, P. T. [2014], 'On the limitations of probability in conceptualizing pattern matches in forensic science: Response to "On the value of probability for evaluating results of comparative pattern analysis" by Alex Biedermann and Franco Taroni [Forensic Sci. Int. 232 (2013) e44-e45]', Forensic Science International 239, e10-e11.

36 [11] Lindley, D. [1985], Making Decisions, second edn, John Wiley \& Sons, Chichester.

[12] Lindley, D. V. [2000], 'The philosophy of statistics', The Statistician 49, 293-337.

38 [13] Lindley, D. V. [2014], Understanding Uncertainty, revised edn, John Wiley \& Sons, Hoboken.

[14] Marquis, R., Schmittbuhl, M., Mazzella, W. D. and Taroni, F. [2005], 'Quantification of the shape of handwritten characters: a step to objective discrimination between writers based on the study of the capital letter O', Forensic Science International 150, $23-32$.

[15] O’Hagan, A., Buck, C., Daneshkhah, A., Eiser, J., Garthwaite, P., Jenkinson, D., Oakley, J. and Rakow, T. [2006], Uncertain judgements : eliciting experts' probabilities, John Wiley \& Sons, Chichester.

[16] Parsons, S. [2001], Qualitative Methods for Reasoning Under Uncertainty, The MIT Press, Cambridge, Massachusetts.

44 [17] Press, S. J. [2003], Subjective and Objective Bayesian Statistics: Principles, Models, and Applications, second edn, Wiley-Interscience, Hoboken.

46 [18] Smith, J. Q. [1995], 'A conversation with Dennis Lindley', Statistical Science 10, 305-319.

[19] Stoney, D. A. [1991], 'What made us ever think we could individualize using statistics?', Journal of the Forensic Science Society 31, 197-199.

48 [20] Taroni, F., Bozza, S. and Aitken, C. G. G. [2005], 'Decision analysis in forensic science', Journal of Forensic Sciences 50, 894-905.

[21] Wellman, M. P. [1990], 'Fundamental concepts of qualitative probabilistic networks', Artificial Intelligence 44, 257-303. 